\title{
Renal cell carcinoma: ten years of significant advances
}

\author{
Olivier Rixe • Brian Rini
}

Received: 3 August 2010 / Accepted: 3 August 2010/Published online: 12 August 2010

(C) Springer-Verlag 2010

Outstanding progress has been made over the past decade for the treatment of metastatic renal cell carcinoma (RCC) [1]. Targeted agents, including antiVEGFs and mTOR inhibitors, have dramatically improved the outcome of metastatic RCC. These drugs have been made a reality through both the recent developments in molecular biology successfully translated to the clinic, and also the initial pertinent observation made by clinicians in the early phase of development of these fascinating new tools.

An essential observation was made during the phase 1 study conducted with an unknown anti-VEGF receptor called SU 11248 [2]. The investigators identified an immediate activity in refractory metastatic RCC based on the occurrence of tumor shrinkage (including complete responses which were quite unusual in phase I studies), but also on the identification of a new phenomenon observed with this class of agents: tumor necrosis. This observation and enthusiasm opened several avenues for the treatment of RCC and created a shortcut for drug development immediately relayed by regulatory agencies in the United States, Europe and all other continents. Based on these

O. Rixe $(\bowtie)$

Experimental Therapeutics Program,

University of Cincinnati College of Medicine,

231 Albert Sabin Way ML 0562,

Cincinnati, OH 45267-0562, USA

e-mail: olivier.rixe@uc.edu

B. Rini

Department of Solid Tumor Oncology,

Cleveland Clinic Taussig Cancer Institute,

9500 Euclid Avenue/Desk R35,

Cleveland, OH 44195, USA

e-mail: rinib2@ccf.org observations and others [3], a new paradigm was established: RCC, considered as a refractory tumor to medical therapies, became a "chemosensitive" tumor to targeted therapies.

If the impact on survival curves for antiangiogenics appears to be "statistically significant" by comparison to conventional therapies, there is a real need to amplify the phenomenon in order to increase the rate of complete responses, duration of response leading to a higher percentage of long-term survivors and ultimately a high cure rate for metastatic disease. This is the reason why the clinicians should step back to the bench to identify with our basic scientist colleagues (molecular biologists, biochemists, imaging researchers, etc.) a smarter direction to use and develop these targeted agents to identify 1 ) predictive factors of drug activity, 2) drug synergies, 3) mechanisms of drug resistance, and 4) optimization in the management of drug toxicities.

In this issue, we have asked our colleagues to use their expertise and knowledge to summarize the enormous acquisition of knowledge in biology (reviewed by Hacker and Rathmell, Vickers and Heng), imaging (van der Veldt et al.), therapy for locally advanced disease (Jonasch and Tannir), drug development in non-clear carcinoma (Singer et al.), and to draw the future direction to reach our ultimate goal and impact survival (Pal and Figlin). We may have reached a plateau in the acquisition of clinical benefits with therapy as currently applied. However, recent advances need to be emphasized and open new perspectives for the development of molecular targeted therapies. For instance, the positive results recently observed with ipililumab in metastatic melanoma have validated the interest for anti-CTLA4 therapy with a positive impact on overall survival [4]. Similar observation has been made in metastatic RCC 
[5], and the use of these new tools in multimodal therapies for RCC is a viable option. In addition, T-cell therapies and the last generation of allogeneic transplantation $[6,7]$ represent fascinating solid perspectives. In this context, the role of molecular therapeutics will be evaluated. In addition, the identification of individualized biomarkers recently published by the Johns Hopkins group using tumor genome sequencing and circulating DNA represent a real advance for the quest of the ideal biomarker [8], with real application for RCC.

Several ongoing trials will provide a clue for the future (extensively reviewed in this issue by Basu and Eisen). For example, the PREINSUT neo-adjuvant study will collect significant cognitive information on both mechanism of action, drug resistance and predictive factors of action for the use of antiangiogenics. This is a fascinating era for medical oncologists, surgical oncologists, biologists and imagers, and hopefully for our patients.

\section{References}

1. Rini BI, Campbell SC, Escudier B (2009) Renal cell carcinoma. Lancet 373(9669):1119-1132

2. Faivre S, Delbaldo C, Vera K et al (2006) Safety, pharmacokinetic, and antitumor activity of SU11248, a novel oral multitarget tyrosine kinase inhibitor, in patients with cancer. J Clin Oncol 24(1):25-35

3. Yang JC, Haworth L, Sherry RM et al (2003) A randomized trial of bevacizumab, an anti-vascular endothelial growth factor antibody, for metastatic renal cancer. N Engl J Med 349(5):427-434

4. Hodi FS, O'Day SJ, McDermott DF et al (2010) Improved survival with Ipilimumab in patients with metastatic melanoma. $\mathrm{N}$ Engl $\mathrm{J}$ Med Jun. doi:10.1056/NEJMoa1003466, Jun 14

5. Yang JC, Hughes M, Kammula U et al (2007) Ipilimumab (anti-CTLA4 antibody) causes regression of metastatic renal cell cancer associated with enteritis and hypophysitis. J Immunother 30(8):825-830

6. Yang JC, Childs R (2006) Immunotherapy for renal cell cancer. J Clin Oncol 24(35):5576-5583

7. http://clinicaltrials.gov/ct2/show/NCT01014234?term=rapamycin\& rank $=5$

8. Leary RJ, Kinde I, Diehl F et al (2010) Development of personalized tumor biomarkers using massively parallel sequencing. Sci Transl Med 2(20):20ra14 